\title{
Bumetanide for neonatal seizures—back from the cotside
}

Ronit M. Pressler, Geraldine B. Boylan, Neil Marlow, Linda S. de Vries, Mats Blennow, Catherine Chiron, J. Helen Cross, Boubou Hallberg, Lena Hellström-Westas, Vincent Jullien, Barry Mangum, Brendan Murphy, Deirdre Murray, Gerard Pons, Janet Rennie, Mona C. Toet and Sarah Zohar for the NEMO consortium (NEonatal seizure treatment with Medication Off-patent)

In a recent News \& Views article (Neonatal seizures still lack safe and effective treatment. Nat. Rev. Neurol. 11, 311-312; 2015), ${ }^{1}$ Thoresen and Sabir reviewed our report on the use of bumetanide as an adjunct to a second dose of phenobarbital in the treatment of neonatal seizures (Bumetanide for the treatment of seizures in newborn babies with hypoxic ischaemic encephalopathy [NEMO]: an open-label, dose finding, and feasibility phase $1 / 2$ trial. Lancet Neurol. 14, 469-477; 2015). ${ }^{2}$ The authors concluded that there might be benefit from bumetanide, but we believe they have misinterpreted our paper and we disagree with their conclusions in several important areas.

The primary outcome in our study was $80 \%$ reduction in seizures-without the need for rescue antiepileptic drugs-arising in $>50 \%$ of infants. Thoresen and Sabir rightly point out that five babies demonstrated seizure reductions of $\geq 80 \%$ of baseline, but rescue drugs for ongoing seizures were used in four of these five within $24 \mathrm{~h}$ of the initial bumetanide treatment period. Nine babies with seizures at baseline were given rescue drugs during the $48 \mathrm{~h}$ after bumetanide treatment. Of the remaining five infants in our sample, who did not experience a seizure by the time bumetanide was administered, three subsequently required rescue medication. Only two babies did not receive rescue treatment, and they did not have seizures at baseline. Thus, any 'success' of bumetanide was temporary, and the prespecified endpoint was not reached by a single patient.

In addition to the primary outcome, we calculated the instantaneous seizure burden in each baby, as measured by seizure intensity over the duration of the observational period. Two independent observers found no evidence that bumetanide altered the immediate trajectory of seizures in any baby; indeed, the seizures were often already decaying in frequency by the time bumetanide was given (Figure 1). Thus, there was no evidence for a direct antiepileptic effect. We could not exclude the possibility that bumetanide enhances the response to rescue drugs - which would require a different study design-however, a new study in mice suggests bumetanide does not improve the effects of phenobarbital in hypoxia-induced seizures, ${ }^{3}$ which supports our findings.

Our trial ${ }^{2}$ used a Bayesian dose-finding approach, ${ }^{4}$ and a separate Bayesian procedure was used to forecast the pharmacokinetics of bumetanide. ${ }^{5}$ We believe that Thoresen and Sabir misunderstood the Bayesian approaches that were used for both the dosefinding and pharmacokinetics methodology: the dose-finding approach does not require a formal sample size calculation, but we did undertake a simulation study to evaluate the robustness of our models. This simulation is described in the web material attached to our original publication. ${ }^{2}$

We designed our trial with predefined stopping rules that required us to halt the study if all dose levels demonstrated toxicity higher than the target level. An acceptable safety profile was defined as serious adverse reactions in $<10 \%$ of patients. Hearing loss is a serious permanent disability, and was seen in three of 11 surviving infants ( 27\%).

Thoresen and Sabir argue that the rate of hearing impairment we observed does not differ from a recent case series to which they contributed, ${ }^{6}$ but this level is substantially higher than was seen in a recent meta-analysis ${ }^{7}$ and in our own audits. In the case series, ${ }^{6}$ the authors conclude that high gentamicin levels contribute to the risk of hearing loss, but they reported only trough gentamicin levels above $2 \mathrm{mg} / \mathrm{l}$. From a

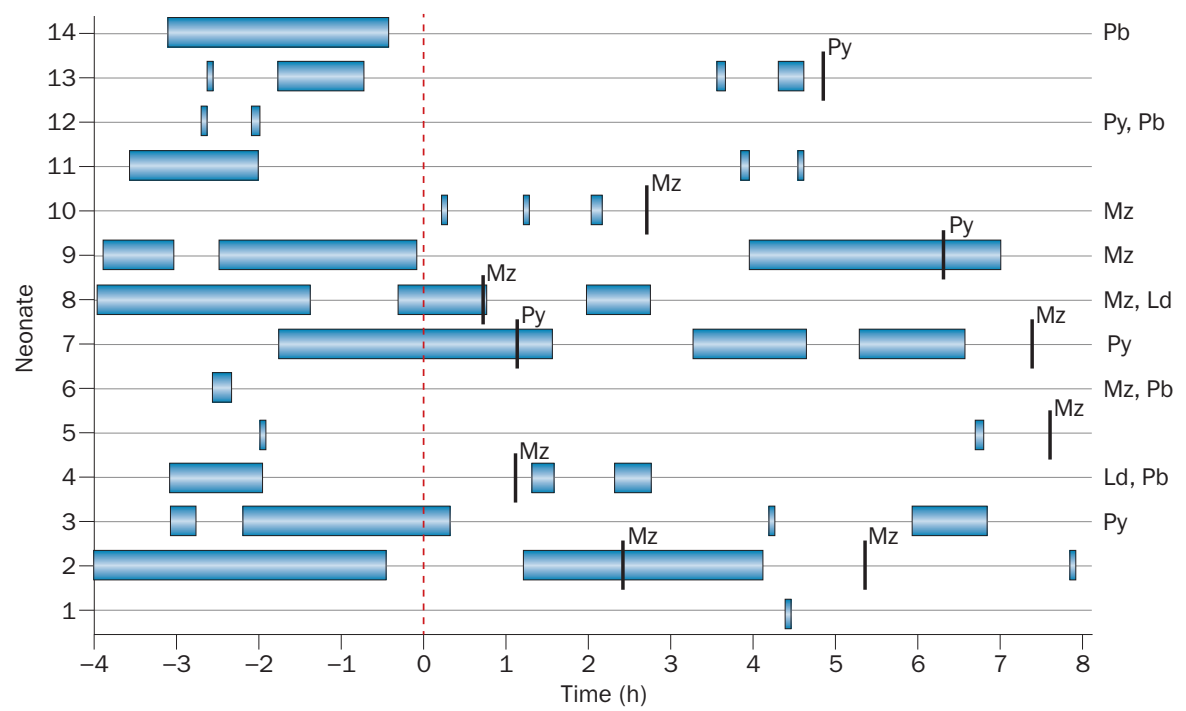

Figure 1 | Instantaneous seizure burden in infants treated with bumetanide. Seizures (blue bars) in all infants from our cohort $4 \mathrm{~h}$ before and $8 \mathrm{~h}$ after the initial bumetanide administration (red dashed line). Bumetanide was given together with a further dose of phenobarbitone, and additional rescue medications (if necessary) are denoted by black lines. Baseline was defined as the 2-h period from -2 to $0 \mathrm{~h}$. Drugs listed to the right of the graph relate to rescue therapies given in hours 9-48. Abbreviations: Ld, lidocaine; Mz, midazolam; Pb, phenobarbital; Py, phenytoin. 
pharmacological perspective, high trough levels are associated with renal dysfunction, whereas high peak levels are associated with ototoxicity. Other data in preterm infants have highlighted a confluence of risk factors including acidosis, loop diuretics and aminoglycosides at target levels. ${ }^{8}$ None of the infants included in our study had either high trough or high peak aminoglycoside levels, or hypoglycaemia. It is plausible that aminoglycosides and loop diuretics (such as bumetanide) act synergistically to produce ototoxicity, ${ }^{9}$ and so extreme caution is required before co-administering these types of drug.

We are also surprised at the authors' statement that "we do not know whether [hypothermia] is also beneficial in humans" in terms of reducing seizure burden. ${ }^{2}$ Recent publications clearly indicate that hypothermia reduces seizure burden in infants with moderate hypoxic-ischaemic encephalopathy. ${ }^{10,11}$

Given the lack of effect of bumetanide on either the primary outcome or the trajectory of seizure burden, and the anxiety concerning the risk of hearing impairment, the NEMO investigators were unanimous in deciding to halt the study. Therefore, we firmly disagree with the conclusion that further clinical studies of bumetanide are warranted in babies with neonatal seizures due to hypoxic-ischaemic encephalopathy.
Section of Clinical Neurosciences \& Neonatal Unit, University College London, 30 Guilford Street, London WC1N 1EH, UK (R.M.P., N.M., J.H.C., J.R.). Neonatal Brain Research Group, INFANT Research Centre, University College Cork, Ireland (G.B.B., B. Murphy, D.M.). Neonatology, Karolinska University Hospital, Sweden (M.B., B.H.). INSERM U1129, France (C.C., V.J., G.P.). University Paris Descartes, France (C.C., V.J., G.P.). Neonatology, University Medical Center Utrecht, Netherlands (L.S.d.V.). Department of Women's and Children's Health, Uppsala University, Sweden (L.H.-W.). Paidion Research, Durham, USA (B. Mangum). Universitair Medisch Centrum Utrecht (UMCU), Netherlands (M.C.T.). Department for Statistics, INSERM, France (S.Z.).

Corresponding author: R.M.P.

r.pressler@ucl.ac.uk

\section{Acknowledgments}

This trial was funded by the European Community's Seventh Framework Programme (FP7-HEALTH2009-4.2-1, grant agreement 241479-The NEMO Project). Data interpretation was the responsibility of the trial steering committee (advised by an independent data monitoring committee). R.M.P. and G.B.B. were joint senior authors for the original trial. ${ }^{2}$

\section{Competing interests}

The authors declare no competing interests.

1. Thoresen, M. \& Sabir, H. Epilepsy: Neonatal seizures still lack safe and effective treatment. Nat. Rev. Neurol. 11, 311-312 (2015).

2. Pressler, R. M. et al. Bumetanide for the treatment of seizures in newborn babies with hypoxic ischaemic encephalopathy (NEMO): an open-label, dose finding, and feasibility phase 1/2 trial. Lancet Neurol. 9, 1474-4422
(2015).

3. Zohar, S. \& O'Quigley, J. Optimal designs for estimating the most successful dose. Stat. Med. 25, 4311-4320 (2006).

4. Kang S. K., Markowitz, G. J., Kim, S. T., Johnston, M. V. \& Kadam, S. D. Age- and sexdependent susceptibility to phenobarbitalresistant neonatal seizures: role of chloride co-transporters. Front. Cell. Neurosci. 9, 173 (2015).

5. Pons, G., Tréluyer, J. M., Dimet, J. \& Merlé, Y. Potential benefit of Bayesian forecasting for therapeutic drug monitoring in neonates. Ther. Drug Monit. 24, 9-14 (2002).

6. Smit, E. et al. Factors associated with permanent hearing impairment in infants treated with therapeutic hypothermia. J. Pediatr. 163, 995-1000 (2013).

7. Jacobs, S. E. et al. Cooling for newborns with hypoxic ischaemic encephalopathy. Cochrane Database of Systematic Reviews, Issue 1. Art. No.: CD003311. http://dx.doi.org/ 10.1002/14651858.CD003311.pub3.

8. Marlow, E. S., Hunt, L. P. \& Marlow, N. Sensorineural hearing loss and prematurity. Arch. Dis. Child. Fetal Neonatal Ed. 82, F141-F144 (2000).

9. Wang, T. et al. Bumetanide hyperpolarizes madin-darby canine kidney cells and enhances cellular gentamicin uptake by elevating cytosolic $\mathrm{Ca}^{2+}$ thus facilitating intermediate conductance $\mathrm{Ca}^{2+}$ activated potassium channels. Cell Biochem. Biophys. 65, 381-398 (2013).

10. Srinivasakumar, P. et al. Therapeutic hypothermia in neonatal hypoxic ischemic encephalopathy: electrographic seizures and magnetic resonance imaging evidence of injury. J. Pediatr. 163, 465-470 (2013).

11. Low, E. et al. Cooling and seizure burden in term neonates: an observational study. Arch. Dis. Child. Fetal Neonatal Ed. 97, F267-F272 (2012). 